\title{
A CASE OF PULMONARY BLASTOMYCOSIS IN A COMMON ELAND (TAUROTRAGUS ORYX)
}

\author{
O. O. ALAKA, T. A. JARIKRE, B. N. OGUNRO, Y. G. GURUMYEN, \\ A. C. MARK, T. O. OMADEVUAYE, B. O. EMIKPE, G. A. ADENIRAN, \\ V. O. TAIWO \& O. B. KASALI \\ Department of Veterinary Pathology, Faculty of Veterinary Medicine, Uni- \\ versity of Ibadan, Nigeria
}

\section{Summary}

Alaka, O. O., T. A. Jarikre, B. N. Ogunro, Y. G. Gurumyen, A. C. Mark, T. O. Omadevuaye, B. O. Emikpe, G. A. Adeniran, V. O. Taiwo \& O. B. Kasali, 2019. A case of pulmonary blastomycosis in a common eland (Taurotragus oryx). Bulg. J. Vet. Med., 22, No 1, $114-121$.

This case report describes for the first time pulmonary blastomycosis in a captive common eland (Taurotragus oryx). The animal has been in captivity for over 14 years and the clinical signs observed before death were non-specific. The carcass was examined grossly and histologically using special stains. There were yellowish, firm and gritty nodules of varying sizes $(0.5-2 \mathrm{~cm}$ in diameter $)$ on the pleura, in the lung, mediastinal lymph nodes and pericardium. The nodules showed pyogranulomatous inflammation and broad based budding yeast (PAS, Giemsa positive and ZN negative) consistent with Blastomyces dermatitidis. Regular screening of soil and environment including animals in captivity should be encouraged to avert possible spread of the mold in favourable conditions. Public awareness should be improved on fungal diseases.

Key words: blastomycosis, eland, Nigeria, pulmonary

Blastomycosis is an infectious noncontagious disease, common to dogs and humans, and seen occasionally in cats, horses and other species (Saccente \& Woods, 2010). Blastomycosis is caused by the dimorphic fungus Blastomyces (Ajellomyces) dermatitidis, which exists as yeast (asexual) in tissues and as mould in nature and at room temperature. The transition from the mould to the yeast phase has been associated with a heat- related partial uncoupling of oxidative phosphorylation (Medoff et al., 1987). It is a chronic pyogranulomatous disease and was first reported by Gilchrist (1898).

Blastomycosis is more prevalent in certain regions of United States, Canada and has been reported in Europe, Africa, and the Middle East (Rodríguez-Tovar et $a l ., 2015)$. It occurs predominantly among humans who live in watershed areas (Baumgardner et al., 2006). This condi- 
tion has been reported in aquatic mammals including the sea lion (Zalophus californianus and Eumetopias jubatus) (Zwick et al., 2000) and dolphins (Tursiops truncatus) (Cates et al., 1986). It has also been reported in wild animals, including the Indian fruit bat (Pteropus giganteus), ferret (Mustela putorius furo), African lion (Panthera leo), American black bear (Ursus americanus) (Storms et al., 2003), wolves (Canis lupus) (Thiel et al., 1987) and coyotes (Canis latrans) (Rodríguez-Tovar et al., 2015). However, it has been rarely observed in ruminant species. This case report describes for the first time pulmonary blastomycosis in common eland (Taurotragus oryx) in a University Zoo, in Nigeria.

Case description. A female eland, (Taurotragus oryx) - one of two, that had been in the collection of the University of Ibadan Zoological Gardens for over 14 years had been losing and regaining body condition in the last one year although regular administration of multivitamins and screening for parasites were usually employed. A few non-specific signs of dullness and inappetence were observed. A day prior to death, the animal was alert with no signs of ill health. The eland was found dead in its pen the following day on sternal recumbency with left lateral flexion of the head and neck with a mucoid discharge from the nasal orifices.

Grossly, the carcass was moderately dehydrated (showing loss of skin turgor, sunken eyes) and emaciated (bony prominences). The ocular and oral mucous membranes were markedly pale. The lower incisors were uneven and severely worn (senile). The lungs were noncollapsed. The pleura were markedly thickened and opaque especially caudodorsally. The pleural surface of the right lung was adherent to the rib cage. There were fibrous tags on the right lung which loosely bound the various lung lobes together. Both lungs were slightly oedematous and moderately congested caudoventrally. There were numerous pale yellowish, firm and mineralised nodules of varying sizes $(0.5-2 \mathrm{~cm}$ in diameter) on the pleura, in the lung parenchyma, mediastinal lymph nodes and pericardium. The nodules were gritty, whitish on incision with a chalky consistency (Fig. 1A). There was serous atrophy of the pericardial, peri renal and coronary fat. The kidneys were pale brown with localised areas of ecchymosis on the cortical surface. Numerous cysts of varying sizes $(3-10 \mathrm{~mm})$ were also observed in the renal medulla. The rumen was filled with partly digested grains and grasses. Within the ruminal content a thick fairly long $(15 \mathrm{~cm})$ ropy twine (foreign body) made of polythene and cotton material was embedded. The abomasal wall was moderately oedematous and the mucosa was diffusely hyperaemic. The meningeal blood vessels were moderately and diffusely congested. No gross lesions were seen in the spleen, liver and intestines.

Cytological examination of Giemsa stained impression smears from mediastinal lymph nodes and lung nodules revealed a few broad based budding yeasts with a suppurative to pyogranulomatous exudate.

Histologically, the lung showed marked thickening of the pleura due to fibrosis and mononuclear cellular infiltrates. There was also marked obliteration of the affected lung parenchyma with diffuse alveolar damage, honey combing of alveoli due to fibrosis of inter-alveolar septae and intra-alveolar fibrous connective tissue proliferation. There were numerous intra-alveolar macrophages and Langhan's giant cells (Fig. 1B) in the al- 

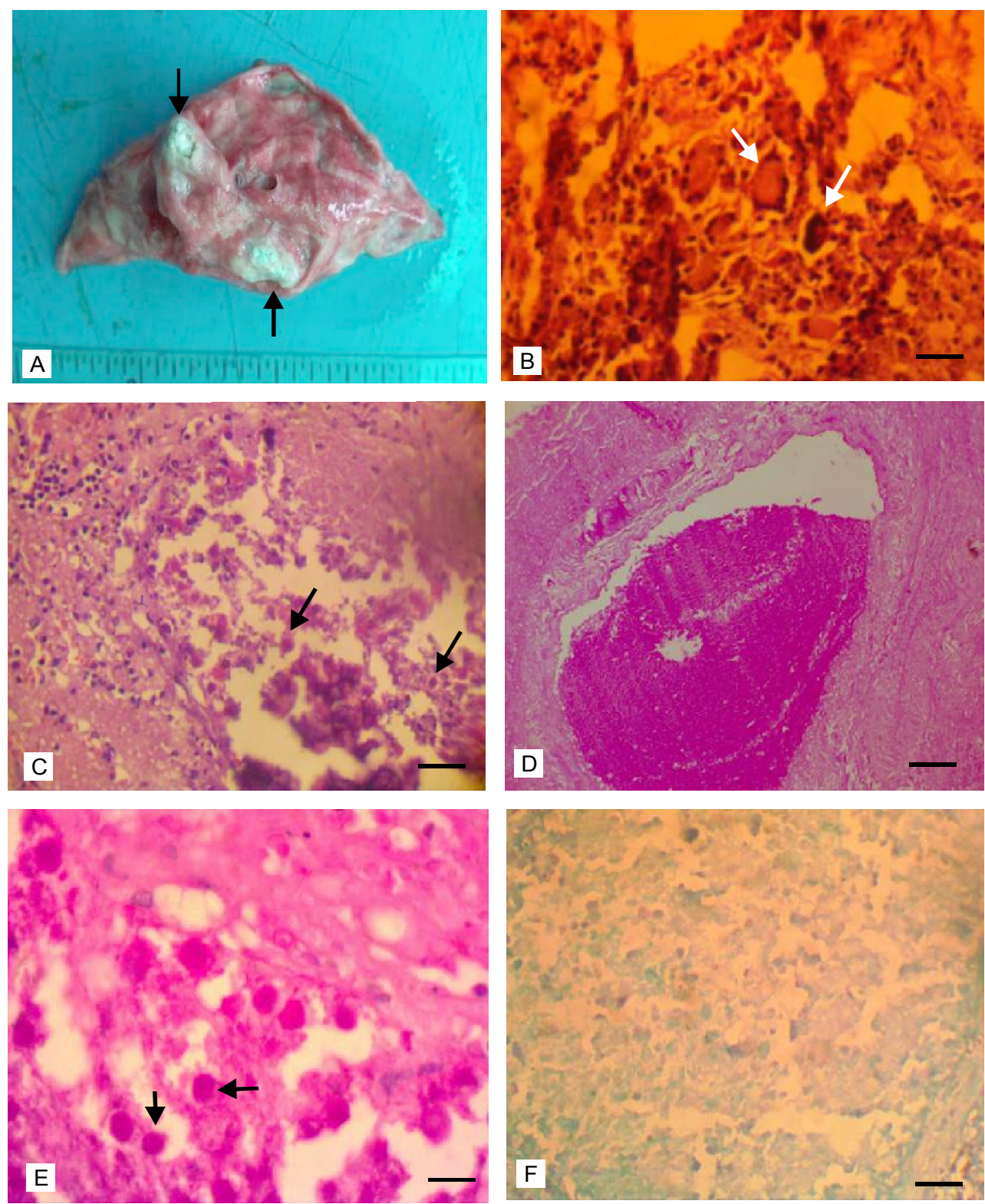

Fig. 1. A. Gritty nodules (arrow) on the pulmonary lobe. B. Granulomatous inflammation with giant cells (arrow) in air spaces (H/E, scale bar $=25 \mu \mathrm{m})$; C. Peribronchiolar cellular infiltrates with intra lesional yeasts (arrow) in airway lumen $(\mathrm{H} / \mathrm{E}$, scale bar $=25 \mu \mathrm{m})$; D. Intensely PAS positive bronchial exudate (scale bar $=25 \mu \mathrm{m}$ ); . E. PAS-stained yeasts (arrows) in bronchial exudate (scale bar $=25$ $\mu \mathrm{m}) ; \mathbf{F}$. The same lung section negative for $\mathrm{ZN}$ stain (scale bar $=25 \mu \mathrm{m}$ ). 
veoli. Bronchi and terminal bronchioles were partially occluded by extensive fibroplasia and inflammatory cellular infiltrates comprising macrophages, neutrophils, lymphocytes, and plasma cells with numerous intra-lesional yeasts (Fig. 1C). There was chondromalacia, mineralisation of necrotic cartilage, alveoli and diffuse fibrosis. Foci of circumscribed pyogranulomatous reactions were within the lung parenchyma. The lymph node showed marked cortical fibrosis and accentuation of the medulla due to follicular hyperplasia and sinus histiocytocysis with numerous giant cells.

Microscopic examination of PASstained lung tissue sections revealed the presence of numerous round yeasts $(10$ $20 \mu \mathrm{m})$ with double contoured retractile wall mixed with necrotic debris within the airway (Fig. 1D, E). The yeast were mostly single with a few broad based. The morphology of the yeast was consistent with that of Blastomyces dermatitidis. Microscopic examination of $\mathrm{ZN}$-stained lung tissue sections did not reveal any acid fast bacilli (Fig. 1F).

This report presents a case of pulmonary blastomycosis in a captive common Eland (Taurotragus oryx). The common eland (Taurotragus oryx) is a species of the family Bovidae that primarily feed on grasses and leaves in savannah. This may be the first case report of this condition in eland and by extension in wild bovine species. Blastomycosis has been reported in canids, humans, equids and aquatic animals; either as pulmonary, systemic or cutaneous forms especially in humans. Pulmonary blastomycosis seems to be more frequent in animals than the disseminated form.

Pulmonary blastomycosis has been diagnosed in wild felids (Storms et al., 2003), rhesus monkey (Macaca mulatta)
(Wilkinson et al., 1999), polar bear (Ursus maritimus) kept in captivity (Morris et $a l .$, 1989), as well as a wild wolf (Thiel et al., 1987) and wild coyotes (RodríguezTovar et al., 2015), however, this is the first report in a common eland (Taurotragus oryx).

The consequences of infection with $B$. dermatitidis are variable and range from subclinical infection to fatal disseminated disease (Saccente \& Woods, 2010). Pulmonary blastomycosis presents clinically as acute pneumonia, chronic pneumonia, or asymptomatically as observed in this case (Patel et al., 1999). The prognosis is usually grave with case fatality rate up to $78 \%$ when infection spreads beyond the lungs (Martin \& Smith, 1939).

The host response to $B$. dermatitidis consist mixed inflammatory cells with clusters of degenerate neutrophils and granulomas, usually noncaseating, with epithelioid and giant cells. Early in the infection, the neutrophilic infiltrates may predominate and organisms easily detected. As granulomas form, the number of organisms tends to decrease.

The lung is the most consistently affected site in animals considering their behavioural and consistent environmental exposures. The lesion is of multifocal to coalescing gray-white nodules, which often range from 3 to $20 \mathrm{~mm}$ diameter (Goldman et al., 1999, Guarner \& Brandt, 2011). Most pulmonary nodules are firm, but some become abscesses or undergo central caseation and then may fistulate into a bronchus or on to the pleura. Mineralisation is minimal or absent. The granulomas are formed by a mass of epithelioid macrophages usually accompanied by histiocytic giant cells and variable numbers of neutrophils, an outer layer of lymphocytes and less reactive macrophages, and often a peripheral rim of fibrous tis- 
sue. In some cases, there is extensive caseous necrosis in the centers of the granulomas, with merely a thin rim of macrophages. Yeast bodies are quite variable in number and may be missed on haematoxylin and eosin-stained sections (Caswell \& William, 2007). The double contour of the fungal wall is well described in cytologic preparations or histologically via special stains as observed in this case.

The skin, soft tissue, bone, genitourinary, or central nervous system involvement have been reported (Lemos et al., 2000; McKinnell \& Pappas, 2009; Bariola et al., 2010). In rare cases, skin lesions without lung involvement have been described, suggesting direct cutaneous inoculation. The skin lesions most frequently described are painless ulcers or verrucous lesions.

The diagnostic tools often employed include thoracic radiography which may show alveolar or mass-like infiltrate (Bradsher et al., 1985; Patel et al., 1999; Patel et al., 2010). However, reticulonodular and miliary opacities and or solitary nodular opacities are also characteristic. This has to be differentiated from tuberculosis, histoplasmosis, and coccidioidomycosis which may present similarly. Special techniques such as polymerase chain reaction (PCR), in situ hybridisation (Hayden et al., 2001) and immunohistochemistry (Fukuzawa et al., 1995, Jensen et al., 1996) could also be helpful, but examination of special stains remains the most reliable and useful method to identify organisms in tissue (Katzenstein, 2006, ElZammar \& Katzenstein, 2007). The auramine-rhodamine stain is also satisfactory, but requires a fluorescent microscope. Periodic acid-Schiff (PAS) Gridley stain is satisfactory but PAS without a counterstain should not be used because organisms are not adequately differentiated from background necrotic debris (Ulbright $\&$ Katzenstein, 1980). The Gomori methenamine silver (GMS) stain is the most useful. Other stains that may be useful for fungi include the Fontana-Masson (FM) and the combined FM-alcian blue, FMmucicarmine, or alcian blue-PAS stains, especially for identifying cryptococci (Lazcano et al., 1991; 1993).

The most significant transmission route for $B$. dermatitidis is inhalation of the conidia. The conidia are aerosolised through disruption of wet soil or organic matter containing microfoci of $B$. dermatitidis mycelia. They are inhaled into the airway of susceptible host for propagation of yeast phase. In dogs, however, intraocular mycosis is the most frequently reported cause of blastomycosis (Broom et al., 1996).

Less commonly, direct cutaneous inoculation via a penetrating outdoor injury, a laboratory accident, or even the bite of an infected dog occurs (Gnann et al., 1983). B. dermatitidis is not transmitted from animals to humans however shared environmental exposures could explain the occurrence of disease in humans especially immunocompromised patients (McKinnell \& Pappas, 2009) and their canine companions (Sarosi et al., 1979).

The source of infection to this eland may be connected to the inhalation of conidia from the contaminated environment. Some environmental factors associated with blastomycosis are sandy acidic soil, bodies of water, and exposure to excavation sites (Arceneaux et al., 1988). Inhalation of infectious spores is considered the main route of pulmonary infection in dogs and is likely the route of infection in this case given the abundance of pyogranulomas and fungal elements in the lungs. Animals often appear to be more susceptible to pulmonary blastomycosis than 
humans because they seem to inhale larger quantities of the fungus spores closer to the ground (Legendre, 2012). Blastomycosis is more prevalent in climatic regions; however, its diagnosis in many animal species and in zoological collections as in this case underscores its importance in Africa.

In conclusion, this is the first reported case pulmonary blastomycosis in a captive eland in Nigeria, this case showed the need for regular screening of soil and environment including animals in captivity to avert possible spread of the mold in favourable conditions hence public awareness on most zoonotic fungal diseases should be improved.

\section{REFERENCES}

Arceneaux, K. A., J. Taboada \& G. Hosgood, 1998. Blastomycosis in dogs: 115 cases (1980-1995). Journal of the American Veterinary Medical Association, 213, 658664.

Baumgardner, D. J., E. M. Knavel, D. Steber \& G. R. Swain, 2006. Geographic distribution of human blastomycosis cases in Milwaukee, Wisconsin, U.S.A.: Association with urban watersheds. Mycopathologia, 161, 275-282.

Bariola, J. R., P. Perry, P. G. Pappas, L. Proia, W. Shealey, W. P. Wright, J. M. Sizemore, M. Robinson \& R.W. Bradsher Jr., 2010. Blastomycosis of the central nervous system: A multicenter review of diagnosis and treatment in the modern era. Clinical Infectious Diseases, 50, 797-804.

Bradsher, R. W., D. C. Rice \& R. S. Abernathy, 1985. Ketoconazole therapy for endemic blastomycosis. Annals of Internal Medicine, 103, 872-879

Broom, J. D., R. E. Hamor \& P. A. Gerding Jr., 1996. Ocular btastomycosis in dogs: 73 cases, 108 eyes (1985-1993). Journal of the American Veterinary Medical Association, 209, 1271-1276.
Caswell, J. L. \& K. J. William, 2007. Respiratory system. In: Pathology of Domestic Animals, vol. 2, $5^{\text {th }}$ edn, eds K. V. F. Jubb, P. C. Kennedy \& N. Palmer, Academic Press, Inc., San Diego, pp. 524-650.

Cates, M. B., L., Kaufman, J. H. Grabau, J. M. Pletcher \& J. P. Schroeder, 1986. Blastomycosis in an Atlantic bottlenose dolphin. Journal of the American VeterinaryMedical Association, 189, 1148-1150.

El-Zammar, O. A. \& A. L. A. Katzenstein, 2007. Pathological diagnosis of granulomatous lung disease: A review. Histopathology, 50, 289-310.

Fukuzawa, M., H. Inaba, M. Hayama, N. Sakaguchi, K. Sano, M. Ito \& M. Hotchi, 1995. Improved detection of medically important fungi by immunoperoxidase staining with polyclonal antibodies. Virchows Archiv, 427, 407-414.

Gilchrist, T. C. \& W. R. Stokes, 1898. A case of pseudolupus caused by Blastomyces. The Journal of Experimental Medicine, $\mathbf{3}$, 53-78.

Gnann, J. W. Jr., G. S. Bressler, C. A. Bodet \& C. K. Avent, 1983. Human blastomycosis after a dog bite. Annals of Internal Medicine, 98, 48-49.

Goldman, M., P.C. Johnson \& G.A. Sarosi, 1999. Fungal pneumonias. The endemic mycoses. Clinics in Chest Medicine, 20, 507-519.

Hayden, R. T., X. Qian, G. D. Roberts, \& R. V. Lloyd, 2001. In situ hybridization for the identification of yeastlike organisms in tissue section. Diagnostic Molecular Pathology, 10, 15-23.

Guarner, J. \& M. E. Brandt, 2011. Histopathologic diagnosis of fungal infections in the $21^{\text {st }}$ century. Clinical Microbiology Reviews, 24, 247-280.

Jensen, H. E., H. C. Schonheyder, M. Hotchi \& L. Kaufman, 1996. Diagnosis of systemic mycoses by specific immunohistochemical tests. Acta Pathologica, Microbiologica et Immunologica Scandinavica, 104, 241-258. 
Katzenstein, A. L. A., 2006. Katzenstein and Askin's Surgical Pathology of NonNeoplastic Lung Disease, $4^{\text {th }}$ edn, Elsevier, Philadelphia.

Lazcano, O., V. O. Speights, J. Bilbao Jr,, J. Becker \& J. Diaz, 1991. Combined Fontana-Masson-mucin staining of Cryptococcus neoformans. Archives of Pathology and Laboratory Medicine, 115, 11451149.

Lazcano, O., V. O. Speights Jr, J. Strickler, J. Bilbao, J. Becker \& J. Diaz, 1993. Combined histochemical stains in the differential diagnosis of Cryptococcus neoformans. Modern Pathology, 6, 80-84.

Legendre, A. M., 2012. Chapter 57. Blastomycosis. In: Greene Infectious Diseases of the Dog and Cat, $4^{\text {th }}$ edn, ed C. E. Green, Saunders, St. Louis, Mo, USA, pp. 606614.

Lemos, L. B., M. Guo \& M. Baliga, 2000. Blastomycosis: Organ involvement and etiologic diagnosis. A review of 123 patients from Mississippi. Annals of Diagnostic Pathology, 4, 391-406.

Martin, D. S. \& D. T. Smith, 1939. Blastomycosis. I. A review of the literature. The American Review on Tuberculosis, 39, 275-304.

McKinnell, J. A. \& P. G. Pappas, 2009. Blastomycosis: New insights into diagnosis, prevention, and treatment. Clinics in Chest Medicine, 30, 227-239.

Medoff, G., A. Painter, \& G. S. Kobayashi, 1987. Mycelial to yeast-phase transitions of the dimorphic fungi, Blastomyces dermatitidis and Paracoccidioides brasiliensis. Journal of Bacteriology, 169, 40554060.

Morris, P. J., A. M. Legendre, T. L. Bowersock, D. E. Brooks, D. J. Krahwinkel, G. M. H. Shires \& M. A. Walker, 1989. Diagnosis and treatment of systemic blastomycosis in a polar bear (Ursus maritimus) with itraconazole. Journal of Zoo and Wildlife Medicine, 20, 336-345

Patel, R. G., B. Patel, M. F. Petrini, R. R. Carter \& J. Griffith, 1999. Clinical presen- tation, radiographic findings, and diagnostic methods of pulmonary blastomycosis: A review of 100 consecutive cases. Southern Medical Journal, 92, 289-295.

Patel, A. J., P. Gattuso \& V. B. Reddy, 2010. Diagnosis of blastomycosis in surgical pathology and cytopathology: Correlation with microbiologic culture. The American Journal of Surgical Pathology, 34, 256261.

Rodríguez-Tovar, L., A. M. Nevárez-Garza, R. V. Barajas-Juárez, J. J. Zarate-Ramos, R. A. Ledezma-Torres \& A. Trejo-Chávez, 2015. Probable pulmonary blastomycosis in a wild coyote (Canis latrans). Case Reports in Veterinary Medicine, DOI $10.1155 / 2015 / 564610$.

Saccente, M. \& L. Woods, 2010. Clinical and laboratory update on blastomycosis. Clinical Microbiology Reviews, 23, 367-381.

Sarosi, G. A., M. R. Eckman, S. F. Davies \& W. K. Laskey, 1979. Canine blastomycosis as a harbinger of human disease. Annals of Internal Medicine, 91, 733-735.

Storms, T. N., V. L. Clyde, L. Munson, \& E. C. Ramsay, 2003. Blastomycosis in nondomestic felids. Journal of Zoo and Wildlife Medicine, 34, 231-238.

Thiel, R. P., L. D. Mech, G. R. Ruth, J. R. Archer \& L. Kaufman, 19870. Blastomycosis in wild wolves. Journal of Wildlife Diseases, 23, 321-323.

Ulbright, T. \& A. Katzenstein, 1980. Solitary necrotizing granulomas of the lung. Differentiating features and etiology. The American Journal of Surgical Pathology, 4, 13-28.

Wilkinson, L. M., J. M. Wallace \& J. M. Cline, 1999. "Disseminated blastomycosis in a rhesus monkey (Macaca mulatta), Veterinary Pathology, 36, 460-462.

Zwick, L. S., M. B. Briggs, S. S. Tunev, C. A. Lichtensteiger \& R. D. Murnane, 2000. Disseminated blastomycosis in two California sea lions (Zalophus californianus). Journal of Zoo and Wildlife Medicine, 31, 211-214. 
O. O. Alaka, T. A. Jarikre, B. N. Ogunro, Y. G. Gurumyen, A. C. Mark, T. O. Omadevuaye ...

Paper received 16.01.2017; accepted for publication 07.04.2017

\section{Correspondence:}

B. O. Emikpe

Department of Veterinary Pathology,

Faculty of Veterinary Medicine,

University of Ibadan, Nigeria

tel: +2348066486080

e-mail:banabis2001@yahoo.com 